\title{
Investigation of the Actual Causes of Hip Joint Implant Loosening Classified as Aseptic - Analysis of Microbiological Culture Results and Levels of Inflammatory Markers
}

\author{
DAGMARA STRZELEC-NOWAK ${ }^{1 *}$, MARIA KOZIOŁ-MONTEWKA ${ }^{1}$, JUSTYNA NIEDŹWIADEK ${ }^{1}$, \\ AGNIESZKA BOGUT ${ }^{1}$, JAN BLACHA ${ }^{3}$ and TOMASZ MAZURKIEWICZ ${ }^{2}$ \\ ${ }^{1}$ Department of Medical Microbiology, Medical University, Lublin, Poland \\ ${ }^{2}$ Department of Orthopaedics and Traumatology, Medical University, Lublin, Poland \\ ${ }^{3}$ Department of Orthopaedics and Traumatology, University of Warmia and Mazury, Olsztyn, Poland
}

Submitted 24 November 2013, revised 21 March 2015, accepted 24 March 2015

\begin{abstract}
Loosening of the hip joint prosthesis is considered as one of the most significant postoperative complications in recent years. The laboratory diagnostic procedure used to differentiate periprosthetic infection from aseptic loosening is very difficult because of the biofilm which microorganisms form on the implant surface. The purpose of this research was to evaluate the level of concordance between clinical classification of implant loosening among 50 patients subjected to reimplantation procedure and laboratory investigation of PJI including microbiological culture results and the levels of inflammatory markers assessed in the patients' synovial fluid samples, serum, and full blood. The synovial fluid was collected for leukocyte count, differential cell count, and culture on standard media. The levels of systemic inflammation markers such as the ESR and CRP concentration were determined in serum and full blood. Tissue samples were collected for microbiological studies. Components from endoprostheses were exposed to ultrasound in a process called sonication. Among the parameters measured in serum and full blood the levels of ESR and CRP were higher in the septic group of patients. Cytologic analysis of synovial fluid was in correlation with microbiologic identification. The most frequent isolated bacteria was Staphylococcus epidermidis. Culture results from materials such as synovial fluid, sonicate and tissues are crucial to establish the infectious aetiology of the loosening. Microscopic analysis of synovial fluid represents a simple, rapid and accurate method for differentiating PJI from aseptic failure. Sonication increases detection of the infectious process, and culture results are in correlation with the cytologic analysis of synovial fluid.
\end{abstract}

Ke y wo rds: aseptic loosening, biofilm, hip joint, implant failure, leukocyte cell count

\section{Introduction}

Hip joint implantations have represented one of the most frequent orthopaedic surgery procedures within the last years. Loosening of the hip joint prosthesis is considered as one of the most significant postoperative complications (Schinsky et al., 2008). It may follow an aseptic or septic course. Aseptic loosening is associated with activation of macrophages caused by wear debris from implant biomaterial which can occur in the periprosthetic space during its normal activity. Septic loosening is a result of the activation of neutrophils and production of inflammatory mediators induced by bacteria. Bacteria which are isolated most frequently include coagulase-negative staphylococci, Staphylococcus aureus, Propionibacterium spp. The causative microorganisms may exist on the implant surface or in its close proximity in forms which significantly hamper their detection by routine cultivation techniques. These forms include the biofilm, SCV (small colony variants) subpopulations or intracellular localization (Marculescu and Cantey 2008; Moran et al., 2010).

Prosthetic joint infections (PJIs) are classified as: early ( $<3$ months after implantation) and late ( $>3$ months after implantation). The early infections are a result of intraoperative bacterial infection whereas the late infections develop as a result of hematogenous bacterial translocation from distant sites of infection. In both cases bacteria can produce biofilm on the implant surface which is considered as one of their most important causes of difficulties associated with treatment of PJIs. Risk factors predisposing to the development of PJIs include previous joint surgeries including their types and numbers, accompanied diseases, age, obesity, rheumatoid arthritis, infections such as urinary tract infections, periodental infections or purulent

\footnotetext{
* Corresponding author: D. Strzelec-Nowak, Department of Medical Microbiology, Medical University of Lublin, Lublin, Poland; e-mail: dagmara.strzelec@gmail.com
} 
skin changes (Górecki et al., 2008; Moran et al., 2010; Zimmerli, 2006).

It should be mentioned that aseptic loosening commonly considered among the most frequent causes of implant dysfunction, has been increasingly reported to be associated with infection. Moreover, aseptic, biomechanical loosening is difficult to differentiate from oligosymptomatic chronic infectious process. It also complicates treatment strategy, which becomes more expensive and not effective, despite reimplantation procedure.

The laboratory diagnostic procedures used to differentiate periprosthetic infection from aseptic loosening include:

- analysis of the levels of CRP, ESR, WBC in patient's serum and full blood,

- white blood cell count and its differential cell counts measured in synovial fluid,

- histopatological examination of periprosthetic tissues based on the determination of the count of neutrophils and macrophages in tissues bioptate,

- microbiological culture of sonicate fluid, synovial fluid, periprosthetic tissue fragments,

- image research - radiography, computer tomography, USG, magnetic resonance, nuclear medicine methods (Bauer et al., 2006; Bedair et al., 2011; Cipriano et al., 2012; Górecki et al., 2008; Moran et al., 2010; Parvizi et al., 2011; Zimmerli, 2006).

According to guidelines published by Parvizi et al. (2011) a definite diagnosis of PJI can be made when the following conditions are met:

1. a sinus tract communicating with the prosthesis or

2. a pathogen is isolated by culture from two separate tissue or fluid samples obtained from the affected prosthetic joint or

3. four of the following six criteria exist:

a. elevated serum erythrocyte sedimentation rate (ESR) or serum C-reactive protein (CRP) concentration,

b. elevated synovial white blood cell (WBC) count,

c. elevated synovial neutrophil percentage (PMN\%),

d.presence of purulence in the affected joint,

e. isolation of a microorganism in one culture of periprosthetic tissue or fluid,

f. greater than five neutrophils per high-power field in five high-power fields observed from histologic analysis of periprosthetic tissue at 400 times magnification (Parvizi et al., 2011).

It should be mentioned that no single routinely used clinical or laboratory test has been shown to achieve ideal sensitivity, specificity, and accuracy for the diagnosis of PJI. Hence, a combination of laboratory, histopathology, microbiology, and imaging studies is frequently necessary (Schinsky et al., 2008; Trampuz and Zimmerli, 2005). The diagnostic significance of the examination of the synovial fluid should be highlighted. Valuable information useful to distinguish between aseptic implant failure and PJI can be provided by the analysis of synovial fluid both macroscopically (its colour, transparency, viscosity) and microscopically (white blood cell count and the polymorphonuclear leukocytes percentage). The obtained results should be interpreted in the context of microbiological culture results (Bedair etal., 2011; Dougados, 1996; Górecki et al., 2008; Zmistowski et al., 2012).

The purpose of our work was to evaluate the level of concordance between clinical classification of implant loosening among patients subjected to reimplantation procedure and laboratory investigation of PJI including microbiological culture results and the levels of inflammatory markers assessed in the patients' synovial fluid samples, serum, and full blood.

\section{Experimental}

\section{Materials and Methods}

Materials were collected from 50 patients (mean age: $71.8+/-10.3$ years) who underwent prosthetic hip joint revision due to loosening between April 2009 and December 2011. The patients were treated at the Orthopaedic Ward of the Clinical Hospital No. 4 in Lublin, Poland. The blood samples were collected in the direct preoperative period. The synovial fluid samples, tissue fragments, and the elements of explanted prostheses were collected intraoperatively. All diagnostic procedures were taken in laboratory of Medical Microbiology Department in Medical University of Lublin, Poland.

In all patients enrolled in the study the loosening was clinically classified as aseptic due to the absence of fistula and/or the purulence in the affected joint. The mean period to the onset of the loosening symptoms was 95 months \pm 62.4 . Eleven patients had undergone previous revision procedures. Among them two patients had a history of two reimplantations whereas the remaining nine experienced one reimplantation. Eight patients suffered from comorbidities such as diabetes, hyperthyroidism, rheumatoid arthritis, chronic renal insufficiency, chronic obstructive pulmonary disease. All investigation was conducted in accordance with the ethical standards of the bioethical committee on human experimentation.

Intraoperatively, tissue samples from the close proximity of the implant and demonstrating the most obvious inflammatory changes were collected for microbiological studies. At least two tissue samples were collected from each patient. The synovial fluid was collected intraoperatively for leukocyte count, differential cell count, and culture from patients with "asep- 
tic" loosening. The prosthetic components were placed in 1-liter, straight-sided, wide-mouthed polypropylene jars that had been autoclaved at $132^{\circ} \mathrm{C}$ for 15 minutes. The specimens were processed by the microbiology laboratory within 2 hours.

The levels of systemic inflammation markers such as the ESR and CRP concentration were determined in the direct preoperative period.

Synovial fluid analysis: Synovial fluid was aspirated intraoperatively using a sterile syringe and transferred to the test-tube containing EDTA. One portion of synovial fluid was analysed in the Fast Read 102 chamber $(40 \times$ magnification $)$ in order to calculate the leukocyte count, the remaining portion was centrifuged $(2500 \times \mathrm{g} / \mathrm{min}$ for 5 minutes at room temperature). The supernatant was frozen at $-80^{\circ} \mathrm{C}$. The sediment was used to prepare a May-Grunwald-Giemsa (MGG) stained smear. The MGG smear was used to establish a percentage of neutrophils, monocytes and limfocytes in the synovial fluid sample (Zimmermann-Górska et al., 1997).

Conventional microbiological methods: Synovial fluid was inoculated in $100 \mu$ laliquots onto aerobic blood agar, chocolate blood agar, and anaerobic blood agar. The aerobic and anaerobic blood agar plates were incubated at $35^{\circ} \mathrm{C}-37^{\circ} \mathrm{C}$ in $5-7 \%$ carbon dioxide aerobically and anaerobically for 7 days.

Tissue specimens were inoculated into thioglycollate broth and incubated at $35^{\circ} \mathrm{C}-37^{\circ} \mathrm{C}$ for up to 7 days. Cloudy thioglycollate broth was subcultured onto conventional bacteriologic media.

Sonication of removed prostheses: Components from endoprostheses were exposed to ultrasounds in process called sonication. Five hundred milliliters of sterile saline were added to each container. The container was vortexed for 30 seconds using a Vortex-Genie and subsequently subjected to sonication for 7 minutes at the temperature of $20^{\circ} \mathrm{C}$ (Monsen et al., 2009). Sonication was followed by additional vortexing for $30 \mathrm{sec}-$ onds. The resulting sonicate fluid was removed under aseptic conditions and placed into 50-ml sterile Falcon tubes. Samples were then centrifuged at $4200 \times \mathrm{g}$ for 20 minutes. One hundred $\mu \mathrm{l}$ of the sedimented sonicate fluid were inoculated onto a set of routine aerobic and anaerobic bacteriologic media. The culture result was considered positive if there were at least 5 colonyforming units of the same organism on either plate.

WBC, ESR and ER analysis: The levels of inflammatory markers in serum and full blood were determined 24 hours before the operation. The WBC counts, serum erythrocyte sedimentation rate in full blood, as well as the CRP concentration in serum were analysed.

Statistical analysis: Statistical analysis was conducted using Statistica programme. In the non-parametric test (U Mann-Whitney) the $\mathrm{p}$ value $<0,05$ was considered statistically significant.

\section{Results}

Positive culture results were obtained from $12 \mathrm{pa}$ tients. The cultivated microorganisms were represented by the following species: Staphylococcus epidermidis, S. aureus, Staphylococcus warneri, Enterobacter cloacae, Streptococcus mitis, Propionibacterium acnes (Table I).

Based on the positive and negative culture results all patients were classified into two groups: aseptic (38 patients) and septic (12 patients). The analysis of the white blood cell count in the synovial fluid and the percentage of neutrophils revealed statistically important difference between aseptical and septical group of patients (Table II). In all septic cases, the white blood cell count and the percentage of neutrophils exceeded the cut-off value: $>1700$ cells $/ \mu$ and $>65 \%$ of polymorphonuclear leukocytes (Trampuz et al., 2007).

There was a statistically significant difference concerning the values of inflammatory markers (ESR, CRP) between aseptic and septic group of patients. The values of these parameters were almost two-fold higher in patients classified as septic than in the group of patients with aseptic implant failure. The mean count of WBC was similar in both groups (Table III).

Table I

The number of aseptical and septical cases of hip joint implant loosening in the aspect of cultured microorganisms

\begin{tabular}{|l|c|}
\hline \multicolumn{1}{|c|}{ Microorganism } & No. of cases \\
\hline Aseptic loosening of the hip & 38 \\
\hline Staphylococcus epidermidis & 6 \\
\hline Staphylococcus warneri & 2 \\
\hline Staphylococcus aureus & 1 \\
\hline Streptococcus mitis & 1 \\
\hline Enterobacter clocae & 1 \\
\hline Staphylococcus epidermidis, Propionibacterium acnes & 1 \\
\hline
\end{tabular}


Table II

Comparison of parameters measured in synovial fluid with the type of implant loosening

\begin{tabular}{|l|c|c|c|c|c|c|c|c|}
\hline $\begin{array}{l}\text { Mean } \\
\text { Type } \\
\text { (SD) } \\
\text { loosening }\end{array}$ & $\begin{array}{c}\text { Synovial } \\
\text { fluid } \\
\text { volume } \\
(\mathrm{ml})\end{array}$ & $\begin{array}{c}\text { White blood } \\
\text { cell count } \\
(\mathrm{cell} / \mu \mathrm{l})\end{array}$ & $\% \mathrm{~N}$ & $\begin{array}{c}\mathrm{PMN} \text { amount } \\
(\mathrm{cell} \mu \mathrm{l})\end{array}$ & $\% \mathrm{M}$ & $\begin{array}{c}\text { Monocytes } \\
\text { cell count } \\
(\mathrm{cell} / \mu \mathrm{l})\end{array}$ & $\begin{array}{c}\text { \%L } \\
\begin{array}{l}\text { Septic loosening } \\
(\mathrm{n}=12)\end{array}\end{array}$ \\
\hline $\begin{array}{l}\text { Aseptic loosening } \\
(\mathrm{n}=38)\end{array}$ & $6.08 \pm 4.5$ & $22.359 .3 \pm 39.868 .5$ & $82.3 \pm 22.9$ & $18.401 .7 \pm 39.493$ & $10.3 \pm 14.8$ & $2.303 \pm 1.846$ & $5.8 \pm 10.5$ & $1.296 .8 \pm 2.183 .6$ \\
\hline $\mathrm{p}$ & $\mathrm{p}>0.05$ & 0.000001 & 0.000025 & 0.000001 & 0.000014 & 0.00001 & 0.001 & 0.0001 \\
\hline
\end{tabular}

Table III

Comparison of gender, time to loosening and inflammatory markers in serum and full blood of patients with septic and aseptic type of implant loosening

\begin{tabular}{|l|c|c|c|c|c|}
\hline Parameter (SD) & $\begin{array}{c}\text { Age } \\
\text { (years) }\end{array}$ & $\begin{array}{c}\text { Time to loosening } \\
(\text { months })\end{array}$ & $\begin{array}{c}\text { ER } \\
(\mathrm{mm} / \mathrm{h})\end{array}$ & $\begin{array}{c}\text { CRP } \\
(\mathrm{mg} / \mathrm{l})\end{array}$ & $\begin{array}{c}\text { WBC } \\
\left(\text { tys } / \mathrm{mm}^{3}\right)\end{array}$ \\
\hline Septic loosening & $68.1 \pm 13.8$ & $82 \pm 70.5$ & $45.7 \pm 24.4$ & $18.49 \pm 20.8$ & $7.67 \pm 3.3$ \\
\hline Aseptic loosening $(\mathrm{n}=12)$ & $71.5 \pm 10.8$ & $78.6 \pm 57.1$ & $27.8 \pm 19.4$ & $10.5 \pm 23.1$ & $6.73 \pm 1.9$ \\
\hline $\mathrm{p}$ & $\mathrm{p}>0.05$ & $\mathrm{p}>0.05$ & 0.004 & 0.004 & $\mathrm{p}>0.05$ \\
\hline
\end{tabular}

Table IV

Time to loosening and inflammatory markers in serum and full blood of patients with different bacterial species

\begin{tabular}{|l|c|c|c|c|}
\hline $\begin{array}{c}\text { Microorganism } \\
\text { (number of cases) }\end{array}$ & $\begin{array}{c}\text { Time to loosening } \\
\text { (months) } \\
\text { SD }\end{array}$ & $\begin{array}{c}\text { ER } \\
(\mathrm{mm} / \mathrm{h}) \\
\mathrm{SD}\end{array}$ & $\begin{array}{c}\text { CRP } \\
(\mathrm{mg} / \mathrm{l}) \\
\mathrm{SD}\end{array}$ & $\begin{array}{c}\text { WBC } \\
\left.\text { (tys/mm } / \mathrm{mm}^{3}\right) \\
\text { SD }\end{array}$ \\
\hline CNS $n=8$ & $79 \pm 72$ & $43.6 \pm 20.4$ & $12.5 \pm 13.1$ & $7.1 \pm 3.5$ \\
\hline S. aureus $n=1$ & 17 & 13 & 13.29 & 13.3 \\
\hline E. cloacae $n=1$ & 169 & 52 & 36.67 & 7.52 \\
\hline S. mitis $n=1$ & 129 & 91 & 72.99 & 7.95 \\
\hline S. epidermidis i P. acnes $n=1$ & 18 & 19 & 0.74 & 7.78 \\
\hline
\end{tabular}

Table V

The synovial fluid inflammatory parameters in the context of cultivated bacterial species

\begin{tabular}{|l|c|c|c|c|c|c|}
\hline $\begin{array}{c}\text { Microorganism } \\
\text { (number od cases) }\end{array}$ & $\begin{array}{c}\text { Synovial fluid } \\
\text { volume (ml) } \\
\text { SD }\end{array}$ & $\begin{array}{c}\text { White blood cells } \\
\text { count (cell/ul) } \\
\text { SD }\end{array}$ & $\begin{array}{c}\text { PMN amount } \\
\text { (cell/ul) } \\
\text { SD }\end{array}$ & $\begin{array}{c}\% \mathrm{~N} \\
\text { SD }\end{array}$ & $\begin{array}{c}\% \mathrm{M} \\
\text { SD }\end{array}$ & $\begin{array}{c}\% \mathrm{~L} \\
\text { SD }\end{array}$ \\
\hline CNS $n=8$ & $4.7 \pm 3.2$ & $5.586 \pm 3.905$ & $4.581 \pm 3.721 .8$ & $75 \pm 25.7$ & $14 \pm 16.9$ & $7.7 \pm 12.3$ \\
\hline S. aureus $n=1$ & 7 & 149.600 & 142.120 & 95 & 0 & 5 \\
\hline S. mitis $n=1$ & 6.5 & 29.915 & 28.120 & 94 & 6 & 0 \\
\hline S. epidermidis i P. acnes $n=1$ & 3.6 & 37.600 & 36.470 & 97 & 3 & 0 \\
\hline E. cloacae $n=1$ & 17 & 6.503 & 6.170 & 95 & 2 & 3 \\
\hline
\end{tabular}

In the next step of the research we investigated the relationship between all parameters measured in the synovial fluid, serum and full blood and the microbial species isolated from synovial fluid, sonicate fluid and periprosthetic tissue cultures (Table IV, Table V).

\section{Discussion}

Despite significant advance in the laboratory and image diagnostics there is still a need to find a simple and fast method of identification of the type of loos- 
ening, soon enough to prevent its progression and to protect the implant. The major criteria used to clinically classify the type of loosening include the RTG image and the results of ER, CRP, WBC analysis in serum and full blood. Microbiologically, culture results from materials such as synovial fluid, sonicate and tissues are crucial to establish the infectious aetiology of the loosening. It should also be mentioned that microscopic analysis of synovial fluid (leukocyte count and differential) represents a simple, rapid and accurate method for differentiating PJI from aseptic failure. The crucial limitation of this diagnostic approach, however, is the invasive (intraoperative) way of collection of this material.

Ideally, the infection is diagnosed (or excluded) before surgery, which enables starting antimicrobial treatment preoperatively and allows planning of the most appropriate surgical management (Trampuz and Zimmerli, 2005).

Synovial fluid, tissues and elements from prosthesis (mandrel, pan) were subjected to microbiological culture. Mandrel and pan were subjected to ultrasounds in a simple and useful process called sonication. The use of sonication increases the chance of dislodging microorganisms adhered to the surface of explanted devices which, in turn, may increase the sensitivity of the culture. Sonication is innovatory method which is very helpful in diagnostic of orthopaedic implant loosening (Monsen et al., 2009; Trampuz et al., 2007).

The results of microbiological culture enabled to classify patients enrolled in the study into two groups: septic (12 cases) and aseptic (38 cases). Bacteria isolated most frequent included S. epidermidis which is an evidence to intraoperatively infection of this skin bacteria (in patients with early type of loosening) or exogenic infection during an operations (in patients with late type of loosening).

The results of bacteriological culture were confronted with cytologic analysis of synovial fluid. This analysis provided early (before the culture results were available) information on the type/nature of the loosening. As mentioned, septic loosening is suspected if at least 1700 white blood cells/ $\mu \mathrm{l}$ and more than $65 \%$ of neutrophils are detected in the synovial fluid sample (Trampuz et al., 2007). Among 11 patients the above mentioned parameters significantly exceed the cutoff values which was indicative of the septic type of loosening. The preliminary diagnosis was confirmed by the presence of microorganisms in 10 synovial fluid microscopic smears and positive culture results from synovial fluid, sonicate fluid and tissues in 12 patients. In 3 out of 50 cases compatibility between the results of microbiological culture and cytological analysis of synovial fluid was not confirmed. In case of one patient from septic group the white blood cell count in synovial fluid exceed the cut-off value whereas the percentage of
PMN was below the cut-off. Nevertheless, this patient was considered as infected due to the positive culture result. In another patient cytological analysis of synovial fluid was not indicative of inflammation associated with infection which stood in contradiction to a positive culture result obtained from the sonicate. We assume that the positive result of culture was a result of material contamination during analysis; therefore, the patient was considered as false-positive. Similarly, in aseptic group, in one patient we cytological analysis of synovial fluid was suggestive of the infectious type of loosening, but the culture result was negative. It could be associated with the short period between the primary surgery and revision - only a few days. The concentration of CRP in the patient's serum was elevated whereas the WBC count remained within the physiological range.

Leukocytosis measured in the synovial fluid seems to be the more useful for the diagnosis of the late type of loosening since high amount of granulocytes shortly after the surgery can be associated with the surgical intervention itself and can remain elevated even for several days (Cipriano et al., 2012).

Taking into account bacterial species and cytologic analysis of synovial fluid important differences concerning polymorphonuclear cells amount between isolated species, which was indicative of the immunological strength of response of macroorganism depending on the pathogen, were observed. The strongest response was generated by $S$. aureus $(149.600$ cells/ $\mu \mathrm{l})$, which represented almost 90 -fold increase of the cutoff value. Other bacteria like S. mitis and S. epidermidis with $P$. acnes also induced strong granulocytic response, equal to 29.915 cells $/ \mu \mathrm{l}$ and 37.600 cells $/ \mu \mathrm{l}$, respectively. Granulocytic response probably depends on the number and types of bacterial virulence factors, that's why it's not a surprise that $S$. aureus causes the strongest reaction of immunological cells.

Synovial fluid analysis includes macroscopic and microscopic observation. The sterile fluid is transparent, clear, yellow, and viscous as an egg white. The cell count is below $100-200$ cells $/ \mathrm{mm}^{3}$, among which monocytes are predominant; other types of cells include neutrophils, lymphocytes, macrophages, synoviocytes and crystals - cholesterol, hydroxyapatites, etc. (Cipriano et al., 2012, Courtney and Doherty, 2009; Zimmermann-Górska et al., 1997). The septic fluid, in turn, is opaque, bloody or purulent, rich in leukocytes (Courtney and Doherty, 2009; Dougados, 1996; Zimmermann-Górska et al., 1997). During the macroscopic analysis of synovial fluid samples collected from patients enrolled in the study opacity and cloudiness was detected in all fluid specimens obtained from infected individuals. Synovial fluid polymorphonuclear granulocytes (PMN) also called the "first line of defense" are characteristic of the septic implant loosening. In addition to their 
accumulation at the site inflammation and antimicrobial effect PMNs send signals to other immune cells in order to control/eradicate the infection. Antimicrobial activity is a result of the presence of granules within neutrophils which contain antimicrobial compounds such as lactoferrin, myeloperoxidase, proteases, elasthases, kathepsin G (Kumar and Sharma, 2010; Leszczyńska-Gorzelak and Poniedziałek-Czajkowska, 2009).

According to literature data, the ESR, CRP and WBC are the inflammatory markers used most frequently to investigate PJIs. Chevillotte and co-workers, Berbari and co-workers or Piper and co-workers also analysed these blood markers. Chevillotte research group reported the above mentioned markers to be non-specific and respresenting a weak diagnostics value. Levels of these parameters may only suggest further directions of the diagnosic proceedings, like the synovial fluid aspiration, blood culture, radiography. Similar conclusions were drawn by KE Piper research group. These authors also detected elevated levels of both ESR and CRP in the septic group of patients compared with aseptic one. According to Berbari's research group the levels of both ESR and CRP remaining within the physiological ranges give the evidence of non-inflammatory nature of the loosening of hip joint prostheses (Berbari,. et al., 2010; Chevillotte et al., 2009; Piper et al., 2010). Our investigations confirmed it in only 17 out of 38 aseptic patients. As mentioned, CRP is a non-specific marker, its concentration can remain elevated during the course of many diseases, eg. heart attack, cancer, and after surgical operations (Leszczyńska-Gorzelak and Poniedziałek-Czajkowska, 2009; Piper et al., 2010; Schinsky et al., 2008). The WBC count, the multifunctional diagnostic parameter, also cannot be associated only with the implant loosening process, which was confirmed in our research. The ESR and CRP also considered as non-specific, can be useful at the first stage of the diagnosis. Patients suffering from PJI had two-fold elevated ESR level compared to the aseptic group of patients. It should also be emphasized that differences in the septic group probably depend on the time to the loosening, because the ESR level is almost 2-fold higher in patients with early type of implant loosening compared to the late one. It was also observed in case of the WBC count, but it can be an effect of short period from the operation.

Comparison of both groups of patients - septic and aseptic revealed that parameters like age, time to loosening, and the WBC count were similar in both groups, with the exception of ESR and CRP, which were statistically significant between both groups noticed. Significant relation between the analysed parameters and bacterial species has not been observed. None of the mentioned markers is not specific only for prosthetic joint infection, additionally, their determination has very low sensitivity. The elevated levels of ESR and CRP may be suggestive of not only PJI but also other health problems like diabetes, kidney disease, heart disease, obesity (Cipriano et al., 2012; Chevillotte et al., 2009; Piper et al., 2010; Schinsky et al., 2008; Shah 2009).

The local immunological response (based on the white blood cell count and the percentage of PMN's) in synovial fluid has higher predictive value in the diagnosis of PJIs. It has been observed that in the septic group these parameters were 70 -fold and 2.5-fold, respectively, higher than in the aseptic group of patients.

Among the measured parameters bacteriological culture result interpreted in the context of cytological analysis of synovial fluid and the levels of ESR and CRP in serum and full blood of patient has the highest diagnostics value for the PJIs identification.

In conclusion we can claim that:

1. Synovial fluid is the most sensitive diagnostic material. It is useful for the preliminary differentiation between septic and aseptic type of implant loosening. 2. Sonication increases detection of the infectious process, and culture results are in correlation with the cytologic analysis of synovial fluid

3. Determination of the levels of ESR and CRP has the highest predictive diagnostic value among inflammatory parameters measured in serum and full blood.

\section{Literature}

Bauer T.W, J. Parvizi, N. Kobayashi and V. Krebs. 2006. Diagnosis of periprosthetic infection. J. Bone Joint Surg. 88A, 4: 869-882.

Bedair H., N. Ting, C. Jacovides, A. Saxena, M. Moric, J. Parvizi, and C. J. Della Valle. 2011. Diagnosis of early postoperative TKA infection using synovial fluid analysis. Clin. Orthop. Relat. Res. 469, 1: 34-40.

Berbari E., T. Mabry, G. Tsaras, M. Spangehl, P.J Erwin, M.H. Murad, J. Steckelberg and D.Osmon. 2010. Inflammatory blood laboratory levels as markers of prosthetic joint infection. J. Bone. Joint Surg. 92, 11: 2102-2109.

Chevillotte C.J., M.H Ali, R.T. Trousdale, D.R. Larson, R.E. Gullerud and D.J Berry. 2009. Inflammatory laboratory markers in periprosthetic hip fractures. J. Arthroplasty 24, 5: 722-727.

Cipriano C.A., N.M. Brown, A.M. Michael, M. Moric, S.M. Sporer and C.J. Della Valle. 2012. Serum and synovial fluid analysis for diagnosing chronic periprosthetic infection in patients with inflammatory arthritis. J. Bone Joint Surg. 94, 7: 594-600.

Courtney P. and M. Doherty. 2009. Joint aspiration and injection and synovial fluid analysis. Best Practice Res. Clin. Rheumatol. 23: 161-192.

Dougados M. 1996. Synovial fluid cell analysis. Baillieres Clin. Rheumatol. 10, 3: 519-534.

Górecki A., W. Marczyński and I. Babiak. 2008. Prophylaxis, identification and treatment of nonspecific infection of bones and joints. (in Polish.) Ortop. Traumatol. Reh. 4, 6, 10: 396-415.

Kumar V. and A. Sharma. 2010. Neutrophils: Cinderella of innate immune system. Int. Immunopharmacol.10: 1325-1334.

Leszczyńska-Gorzelak B. and E. Poniedziałek-Czajkowska. 2009. Inflammatory symptoms: immunological response, local and syste- 
mic symptoms, laboratory values. (in Polish). Zakażenia $w$ położnictwie i ginekologii Chapter 4: 32-44.

Marculescu C.E. and J.R. Cantey. 2008. Polymicrobial prosthetic joint infections. Clin. Orthop. Relat. Res. 466, 6: 1397-1404.

Monsen T., E. Lövgren, M. Widerström and L. Wallinder. 2009. In vitro effect of ultrasound on bacteria and suggested protocol for sonication and diagnosis of prosthetic infections. J. Clin. Microbiol. 47, 8: 2496-2501.

Moran E., I. Byren and B.L. Atkins. 2010. The diagnosis and management of prosthetic joint infection. J. Antimicrob. Chemother. 65, 3: 45-54.

Parvizi J., B. Zmistowski, E.F. Berbari, T.W. Bauer, B.D. Springer, C.J. Della Valle, K.L. Garvin, M.A. Mont, M.D. Wongworawat and C.G. Zalavras. 2011. New definition for periprosthetic joint infection. J. Arthroplasty 26, 8: 1136-1138.

Piper K., M. Fernandez-Sampedro, K.E. Steckelberg, J.N. Mandrekar, M.J. Karau, J.M. Steckelberg, E.F. Berbari, D.R. Osmon, A.D. Hanssen, D.G. Lewallen, R.H. and others. 2010. C-reactive protein, erythrocyte sedimentation rate and orthopedic implant infection. PLoS ONE 5, 2: e9358.

Schinsky M.F., C.J. Della Valle, S.M. Sporer and W.G. Paprosky. 2008. Perioperative testing for joint infection In patients under- going revision total hip arthroplasty. J. Bone Joint Surg. 90, 9: 1869-1975.

Shah K., A. Mohammed, S. Patil, A. McFadyen, and R.M.D. Meek. 2009. Circulating cytokines after hip and knee arthroplasty. Clin. Orthop. Relat. Res. 467, 4: 946-451.

Trampuz A. and W. Zimmerli. 2005. Prosthetic joint infections: update in diagnosis and treatment. Swiss Med. Wkly 135, 17-18: 243-251.

Trampuz A., K.E. Piper, M.J. Jacobson, A.D. Hanssen, K.K. Unni, D.R. Osmon, J.N. Mandrekar, F.R. Cockerill, J.M. Steckelberg, J.F. Greenleaf and others. 2007. Sonication of removed hip and knee prostheses for diagnosis of infection. N. Engl. J. Med. 16, 357, 7: 654-663.

Zimmerli W. 2006. Prosthetic-joint-associated infections. Best Pract. Res. Clin. Rheumatol. 20, 6: 1045-1063.

Zimmermann-Górska I., G. Białkowska-Puszczewicz and M. Puszczewicz. 1997. Analysis of synovial fluid (in Polish). Skrypt dla studentów Oddziału Analityki Medycznej, Poznań.

Zmistowski B., C.Restrepo, R. Huang, W.J. Hozack and J. Parvizi. 2012. Periprothetic joint infection diagnosis. A complete understanding of white blood cell count and differential. J. Arthroplasty 27, 9: 1589-1593. 
